\title{
Sex differences in response to emotion recognition training after traumatic brain injury
}

Duncan R. Babbage, PhD

Associate Professor, Auckland University of Technology, Centre for Person Centred Research Director, Auckland University of Technology, Centre for eHealth

Auckland, New Zealand. ORCID: 0000-0002-9259-9246

Barbra Zupan, $\mathrm{PhD}$

Associate Professor, Central Queensland University, School of Health, Medical and Applied Sciences, Department of Exercise and Health Sciences

Rockhampton, QLD, Australia. ORCID: 0000-0002-4603-333X

Dawn Neumann, $\mathrm{PhD}$

Associate Professor and Research Director, Indiana University School of Medicine, Department of Physical Medicine and Rehabilitation and the Rehabilitation Hospital of Indiana

Barry Willer, $\mathrm{PhD}$

Professor, State University of New York at Buffalo, Jacobs School of Medicine and Biomedical Sciences, Department of Psychiatry

Buffalo, NY. ORCID: 0000-0002-9586-8448

Reprint requests to Duncan Babbage, $\mathrm{PhD}$.

\author{
Corresponding author \\ Duncan Babbage, $\mathrm{PhD}$ \\ duncan.babbage@aut.ac.nz \\ +6499219999 x8145
}

\section{Disclosure}

There is a possibility the software package that delivers the intervention described in this study may be released in the future through a publishing company to enable dissemination of the intervention into clinical practice. If this occurs, authors of the article may receive royalty payments associated with sales of the software.

This is the author's manuscript of the article published in final edited form as:

Babbage, D. R., Zupan, B., Neumann, D., \& Willer, B. (2018). Sex differences in response to emotion recognition training after traumatic brain injury. Brain Injury, 32(12), 1492-1499. https://doi.org/10.1080/02699052.2018.1497811 


\begin{abstract}
Objective: To examine sex differences in the effectiveness of a Stories intervention for teaching affect recognition in people with a traumatic brain injury (TBI).

Setting: Post-acute rehabilitation facilities.

Participants: 203 participants (53 women and 150 men) with moderate to severe TBI were screened. 71 were eligible and randomized to one of three treatment conditions: two affect recognition conditions and an active control (cognition). This paper examines sex differences between the Stories intervention ( $\mathrm{n}=23,5$ women and $18 \mathrm{men})$ and the cognitive treatment control ( $\mathrm{n}=24,8$ women and 16 men).
\end{abstract}

Design: Randomized controlled trial with immediate, 3 month and 6 month follow-up post-tests. Interventions were nine hours of computer-based training with a therapist.

Measures: Facial Affect Recognition (DANVA2-AF); Emotional Inference from Stories Test (EIST).

Results: A significant treatment effect was observed for the Stories intervention for women, who demonstrated and maintained improved facial affect recognition. In contrast, males in our sample did not benefit from the Stories intervention.

Conclusion: This positive finding for the Stories intervention for females contrasts with our conclusions in a previous paper, where an analysis collapsed across sex did not reveal an overall effectiveness of the Stories intervention. This intervention warrants further research and development. 
Sex Differences in Emotion Recognition Training 3

\section{Key Words}

emotion recognition; sex; affect; traumatic brain injury; RCT; treatment 


\section{INTRODUCTION}

Emotional functioning is a key part of human experience, with the capacity to recognize and respond to the emotions of others a core part of this. There is now a large body of evidence that indicates social and emotional functioning varies between women and men [1], which is further supported by an even wider body of research illustrating sex differences in brain functioning that are "pervasive and robust" in both healthy populations and in pathological conditions [p. 2246; 2]. While the neurobiology of sex differences in emotion processing is complex and nuanced [3], differential brain activation patterns have been observed in men and women in response to stimuli that depict emotion [1,3,4], with (relatively subtle) differences appearing early and consistently in course of development [5] and continuing through the lifespan [6]. Typically, women in the general population have been shown both to be better at recognizing emotional cues, and to express themselves more easily, while men display greater responses to potentially threatening stimuli [1]. See [1] for a comprehensive review. There remains, however, a 'paucity' of data on these important issues [7].

One of the hallmark effects of traumatic brain injury (TBI) is disruption to social functioning. This difficulty is partly underpinned by problems in a range of emotion recognition and social cognition capacities [8], including difficulty identifying emotions from faces $[9,10]$, other nonverbal cues [11-13], and from context [14-16], all of which are often significantly compromised.

While sex differences in emotion perception and social functioning have been relatively well documented in the general population, we have much more limited data on such sex differences after brain injury. Where such data is available, sex differences have not always been documented in all areas of functioning relevant to social cognition and communication. For 
instance, moderate to severe impairment in theory of mind has been consistently demonstrated after acquired brain injury, but sex has not been supported to be a moderating variable in this area [17], at least to date. However, in the related area of self-perceived pragmatic communication capabilities [18] some research examining sex differences found that males under-estimated the extent of their difficulties compared to informant ratings - a trend not seen in women - suggesting men may have less awareness of the extent of their own difficulties in this area after brain injury. Similarly, some studies have found men to be more impaired in emotion recognition from faces and in emotional inferencing from context [19]. It has recently been suggested that female sex may be a protective factor against emotion recognition impairment after traumatic brain injury [20]. However, while some studies have found female advantage on a range of dimensions, others have not, and sex differences that may exist in these domains of functioning after traumatic brain injury have not been well characterized [19].

For both men and women, development of interventions that target relearning such social and emotional skills after brain injury are a high priority [21]. Deficits in emotion perception are related to more than solely impaired general cognition [22] - the ability to use nonverbal cues [23], make accurate interpretation of facial and vocal expressions [24] and infer the likely emotional state of others from contextual information are all important [25-27] and are also frequently impaired after brain injury. For people with TBI, such deficits are associated with both functional difficulties and poor long term social outcomes [21,28-30].

Given the prevalence $[9,31]$ and potential impact of emotion recognition deficits on psychosocial functioning, more research is needed on the development and identification of effective interventions targeting these skills in the TBI population [10,11,21,32-34]. A series of small studies have examined treatments designed to improve emotion perception after brain 
injury, most showing positive treatment effects. See Cassell et al. [35] for an excellent review and discussion. However, these studies have largely had small sample sizes (ranging from 3-19 people treated) and methodological limitations, including in particular the lack of a true placebocontrol intervention $[21,33,36]$. To address this, we previously reported outcomes of an international randomized controlled trial of two novel interventions for emotion recognition difficulties after traumatic brain injury, which we compared to an active sham-treatment control intervention. We randomized 71 eligible participants to treatment. One intervention trained emotional inferencing from stories describing relevant contextual information - the Stories intervention — while the other intervention was aimed at improving affect recognition from static facial expressions - the Faces intervention. Primary outcomes were examined immediately following treatment, and at 3 and 6 months post-treatment with measures of facial affect recognition and emotional inferencing from stories [37]. In our paper reporting the primary outcome of that trial, it was concluded that participants who received the Faces intervention significantly improved their facial affect recognition ability, and that this improvement was maintained through to the final six-month follow-up [37]. No significant change was found for those receiving the Stories intervention when the group was examined as a whole compared to the Control group. However, planned subsequent subgroup analyses were planned to examine sex differences in response to treatment for a later date.

Notably, in our former publication of our primary outcomes we discovered that there was a significant sex difference between the three treatment groups $(P=.04)$, with more males (only one female) randomized to the Faces intervention [37]. To determine whether we should control for such demographic variables in the analysis in that paper, we examined potential relationships between our measure of facial affect recognition and a story-based emotional inferencing test 
with sex, education, time post-injury and depression. The facial affect recognition measure was not significantly associated with any of these variables $(r=.001$ to $.111 ; P=.40$ to .99$)$. The story-based emotion inference test was only associated with $\operatorname{sex}(r=.276 ; P=.02)$ and depression at baseline $(r=-.239, P=.045)$, but not the others $(r=-.239$ to $.210 ; P=.11$ to .50$)$. Because the strength of these correlations was so small [i.e., in the range $r=.26$ to $.49 ; 38$ ] these variables were not used as covariates when analyzing the overall findings in the original paper.

Following our original intent to conduct subgroup analyses on sex differences, the objective of the further analysis in the current paper was to examine whether participant sex might be a determinant of treatment response. In the original trial, a total of 203 participants were screened for the full randomized controlled trial and 71 were randomized, including 14 women. It should be noted that in our original design we had not blocked by sex in our randomization, which resulted in chance allocation of almost all of the females in our sample between the Stories intervention ( 5 females alongside 18 males) and to our Cognitive Control ( 8 females alongside 16 males). Just one woman (with 23 males) was randomized to the Faces intervention. With only a single female participant randomized to the Faces intervention, statistical analysis of the contribution of sex to the outcome of that intervention vs. the control condition would be inappropriate. Therefore this paper examines and compares outcomes for females and males on the Stories intervention versus our active cognitive control. Our primary outcome of interest was change scores from each individual's baseline score to their posttreatment average - in particular the difference in these change scores after treatment for control participants versus those who had received the Stories intervention. In the absence of specific data indicating sex differences in responsivity to affect recognition training, rather than simply sex differences in baseline level of functioning, we therefore hypothesized that we would see no 
difference in response to treatment in our female participants compared to male participants for the Stories intervention.

\section{METHODS}

\section{Design}

An international, multi-center randomized placebo-controlled trial comparing two active affect recognition interventions to a sham-control intervention at three post-treatment sessions. Research ethics committees from all participating institutions/regions approved this study.

\section{Participants}

Participants must have sustained a moderate to severe TBI (Glasgow Coma Scale at injury $\leq 12$, posttraumatic amnesia $\geq 24$ hours, or loss of consciousness $\geq 24$ hours). Participants were 18-65 years old, at least 1-year post-injury, and demonstrated sufficient comprehension at screening. Additionally, participants had to have impaired facial affect recognition at screening and maintain this at pre-test (18-28 days later). Exclusion criteria were TBI prior to eight years of age (emotion recognition skills are not sufficiently developed before this age); premorbid developmental or acquired neurologic disorder; premorbid major psychiatric disorder; impaired vision and/or hearing; and substance dependence at the time of study participation. Participants were recruited from the Carolinas Rehabilitation TBI Model Systems Database in North Carolina, rehabilitation facilities and support groups throughout North and South Carolina, southern regions of Ontario, Canada, and in Wellington and Palmerston North, New Zealand. As noted above, a total of 203 participants (including 53 women) were screened for the full randomized controlled trial and 71 were randomized including all 14 women. In particular, 23 participants were randomized to the Stories intervention ( 5 females alongside 18 males) and 24 participants to our Cognitive Control (8 females and 16 males). Among participants randomized 
for treatment, males and females were not significantly different in age, level of education, injury severity, age at injury, or length of time since injury (all $\mathrm{p}>.05$; see Table $\mathrm{S} 1$ in Supplemental Online Materials for means, standard deviations, and F statistics.) Ninety-three percent of randomized participants completed treatment. Retention for those who completed treatment was $98 \%$ at immediate post-test, $91 \%$ at 3 months post-test, and $83 \%$ at 6 months post-test. A detailed description of the recruitment and selection processes is reported in the paper that previously presented the main results of this trial, along with detailed information about participant characteristics, a CONSORT diagram and full discussion of retention [37]. See also further discussion below.

\section{Measures}

This paper presents analysis of primary outcome variables only, to examine any sex differences in primary treatment outcomes. Those measures are outlined below. Participants were also administered a wider group of tests to evaluate cognition, emotion recognition, mood, community integration, neurobehavioral functioning, empathy, relationship support, and hyposmia (diminished sense of smell). For further details see the initial outcomes paper from the trial [37].

Diagnostic Assessment of Nonverbal Accuracy 2-Adult Faces [DANVA2-AF; 39]: The DANVA2-AF is a standardized assessment of facial affect recognition with age-related norms, that has shown appropriate psychometric properties [39-42] and been used with TBI previously $[33,41]$. Twenty-four static faces were displayed on the computer for 15 seconds and participants had to identify the expressed emotion from a list: happy, sad, angry, or fearful. We increased the normal presentation time of 2 seconds to 15 seconds because we did not want to confound our 
results with speed of processing deficits. The DANVA2-AF was also used to determine inclusion (impaired facial recognition).

Emotional Inference from Stories Test (EIST; Zupan, Neumann, Babbage, \& Willer, 2015): The EIST was developed by the authors to measure participants' ability to infer emotions from written contextual information. Stories were presented on a computer visually and auditorily (simultaneously). After a single presentation of each story, participants selected from a list which emotion the main character was feeling: happy, sad, angry, or fearful. (See Zupan et al., 2015, and Neumann et al., 2014, for a discussion of the two variations of this measure used in the study.)

\section{Interventions}

Interventions were a one-on-one computer-assisted treatment facilitated by a therapist, who received approximately 16 hours of training in administering the intervention. Therapists had either completed or were currently enrolled in a graduate-level healthcare-related program; and/or had experience working with patients with TBI. Therapists who administered the treatment were always different from the research assistants who tested the participants. The intervention was primarily administered as nine 1-hour sessions, three times a week, for three weeks. All participants completed 9 hours of treatment guided by a therapist within a 2-3 week

timeframe. A brief description of the two treatments that are the focus of this paper follows-see Neumann et al. [37] for more detailed descriptions of each treatment.

Stories intervention: The Stories intervention taught participants to infer emotions from contextual information presented in short stories. The Stories intervention was developed by the study authors and was piloted in a previous study [33]. The Stories intervention used three main learning concepts: 1) to attend to relevant contextual information provided in the stories and 
associate these with specific emotions; 2) to increase awareness of one's own emotions through introspection and imitation so participants could use their emotional experience to better recognize others' emotions; and 3) to develop associative knowledge and a better conceptual understanding of emotions. A total of 14 short stories were simultaneously presented visually and auditorily on the computer. At the end of each story, participants were asked to identify characters' emotions using story context. Difficulty was gradually increased throughout treatment by using contextual information of progressive subtlety and using vanishing cues.

Cognitive Training Control: This was the sham-treatment control, the purpose of which was to control for the one-on-one attention and person interaction participants in the treatment groups were receiving, without providing any type of emotion-related training. Participants in Cognitive Training played a variety of on-line, publically available computer games that targeted speed of processing, visual scanning, attention, memory, reasoning, and problem-solving skills.

\section{Procedures}

For brevity and to avoid repetition, only an overview of trial procedures is provided here. At screening, and again at pre-test 18-28 days later, participants were administered the DANVA2-AF and EIST, alongside other measures. After the pre-test session, eligible participants were randomized to one of the interventions. Participants were provided the relevant treatment as outlined above. They were then re-assessed at three further points: within four days of completing treatment; 3 months post-treatment; and 6 months post-treatment. See Neumann et al. [37] for fuller details of trial procedures.

\section{Data Analyses}

To conduct our planned subgroup analysis by sex, we characterized any observed sex differences by examining the performance of both males and females in each of our treatment 
conditions. We computed a Mixed MANCOVA outcome analysis for the Stories intervention vs. control group compared by sex, examining the effectiveness of that intervention, and examining potential sex $\times$ time, sex $\times$ group, and $\operatorname{sex} \times$ group $\times$ time interaction effects in our model. Finally, means and standard deviations of intervention group performances by sex for our primary measures are presented across each of the key assessment points of the study.

As age-standardized scores were available for the DANVA2-AF, those scores were converted to $z$-scores prior to analysis. An alpha level of .05 was set for all analyses. Screening and pre-test scores on our outcome measures were averaged into one baseline score for each measure. As previously [37], this baseline score was an additional within-participants covariate in examining effectiveness of the Stories intervention versus Control across the three post-test assessment points for each dependent variable. Data from all participants was analyzed on an intent-to-treat basis. Multiple imputation was used to control for the effects of missing data. Missing data on these measures was almost entirely a result of participant drop-out during the trial. Of particular relevance here is the retention of women in the Stories and Control groups, given small participant numbers in those groups. All five of the women who received the Stories intervention returned for post-test and follow-up at three months. (Males receiving Stories intervention: 17/18 returned at posttest, 14/18 three month follow-up.) Likewise, five women returned at post-test and follow-up for the Control intervention, though this was out of eight originally randomized to that group. (Male controls: 16/16 at post-test, 15/16 at three month follow-up.) At the six month follow-up, only two of the five women in the Stories intervention returned, alongside four of the eight women in the control intervention. (Males: 15/16 for Stories and 15/16 for control at six month follow-up.) These missing individuals were retained in the analysis using multiple imputation. Fifty imputation datasets were generated. Our previous paper 
[37] discusses parameter estimates and pooling procedures. SPSS v.22.0.0.0 and v.23.0.0.2 were used to conduct the analyses reported in this paper.

\section{RESULTS}

\section{Primary Outcomes}

Using a mixed design MANCOVA, we examined the effectiveness of the Stories intervention compared to our Control condition on each of our two primary outcome measures across three post-treatment assessment points (post-test, 3 and 6 month follow-ups), using baseline scores on the measure as a covariate. Table 1 presents the full test statistics for these analyses. For both analyses, Mauchly's tests indicated we did not have reason to reject the null hypothesis of sphericity, and therefore met the necessary assumptions to use MANCOVA.

\section{Diagnostic Assessment of Nonverbal Accuracy-2 Adult Faces}

This analysis indicates a significant interaction effect between group (Stories intervention vs. Control) and sex on the DANVA2-AF ( $\mathrm{P}=.038$; see Table 1). That is, a significant treatment effect was observed for the Stories intervention, and this treatment effect was maintained over the post-treatment time periods, but there was a significant difference in the treatment responses of males and females in our sample to this intervention. There was no main effect of time across the post-treatment assessments, and no interaction between group and time, or sex and time.

-INSERT TABLE 1 ABOUT HERE-

\section{Emotional Inference from Stories Test}

On the EIST, a significant main effect of time was observed $(\mathrm{P}=.001)$, as previously reported in our original outcome analyses, but no significant effect of treatment group nor any interaction effects between group, sex and time. The Stories intervention did not lead to a significant treatment effect compared to the control condition on the EIST. 


\section{Group Means and Standard Deviations}

Mean scores for the Stories group and control condition by sex are reported in Table S2 in Supplemental Online Materials, for each of baseline, post-treatment, and the three and sixmonth follow-up periods.

\section{Observed Pattern of Results}

Figure 1 displays the statistically described relationships between sex and mean outcome scores at each of the assessment points, by intervention group and sex. As confirmed in the statistical analysis, clear differences are apparent for the women of facial affect recognition on the DANVA2-AF in the Control condition vs. the Stories intervention. In contrast, no sustained difference is apparent between men receiving the Stories intervention and males in the Control condition. While females in the Stories group had a superior post-treatment improvement compared to females in the Control group, we also observed a major change in posttest performance among women in the Control condition. This trend was not observed in male controls, raising the possibility that there may be a much stronger test-retest learning effect for women on the DANVA2-AF compared to men. Secondly, mean scores among the five women who received the Stories intervention appear differentiated from the performance of the eight women in the control condition, and appear different also from males treated in either of these two conditions. This is a substantially different pattern to that previously reported in our earlier outcome analysis, and is a difference that clearly underlies the significant interaction effect between group and sex we reported above.

\section{DISCUSSION}


In our original report of outcomes following this randomized controlled trial we reported a positive finding for the Faces intervention on the DANVA2-AF, our primary outcome measure, but we concluded at that time the Stories intervention appeared to be ineffectiveparticipants in the Stories intervention did not perform significantly better than participants in the Control Intervention on any of our outcome measures [37].

In contrast, the current findings provide a different picture, suggesting that females respond to the Stories intervention with improved facial affect recognition abilities, and that these changes are significantly different to those observed in control participants. Our data suggest that this treatment effect could potentially be maintained over six months post-treatment, though this possible conclusion is limited by our six month data being based on just two retained female participants. The finding is consistent with the findings of our pilot study that preceded the currently reported clinical trial, where participants who received the Stories intervention showed significant improvement on a measure assessing their ability to infer and label how they would feel given a hypothetical scenario [33]. In the current study, our closest measure to that domain of functioning is the EIST. However, we did not find a detectable treatment effect on that measure in the current sample, and this included not finding a treatment effect on this measure for women. As noted in our former publication, while facial affect recognition impairments was an eligibility criteria, scores on the stories test was not an eligibility requirement because no standard score was available to determine impairment on the EIST. This measure was developed for the current research, and it is possible it was not sensitive to the intended construct of interest.

Impairment in emotion recognition and social cognition is well documented after brain injury [9], with an encouraging body of research pointing to these deficits as amenable to 
treatment [35], even if there is much work to be done to determine the most effective and lasting approaches to this rehabilitation. As discussed in the introduction of this paper, there is a growing body of convincing evidence that sex differences exist in emotion recognition functioning $[1,3,4]$, that these begin early in the lifespan [5], and continue throughout life [6]. Building on the recent suggestion that sex may be a protective factor from development of emotion recognition difficulties after brain injury [20,44], and may affect responsivity to treatment in other domains such as executive functioning [44], our analysis suggests that sex may also play a role in responsivity to treatment for emotion recognition difficulties.

Of great interest for future research, therefore, is to further elucidate the mechanisms that underpin generalization from training in recognizing emotions through contextual cues, to demonstrating improved performance on a measure of facial affect recognition in photographs, with particular attention to sex differences. As described earlier, our Stories intervention employed three learning strategies: 1) attend to relevant contextual information (e.g., characters' beliefs, expectations, actions); 2) generation and introspection of personal emotional experience (e.g., what an emotion feels like); and 3) creating a deeper associative knowledge regarding emotions. Future research could examine which aspects of the training facilitated female participants' ability to generalize this affective knowledge to facial expressions. Learning which strategies most facilitated the outcomes for females may provide more insight into the underlying mechanism contributing to the generalization of training focused on contextual information to recognition of facial emotion expressions. Perhaps males could also generalize training if provided with different strategies, or if given a more explicit association between the various affective cues. Determining what potential advantages females may have had that facilitated their generalization of affective information from context to faces would be enlightening. 
In our original outcome report, we recommended that the Stories intervention not be dismissed prematurely and continue to be studied, as the ability to anticipate and label one's own emotions, a key mechanism targeted in this treatment arm, is an important precursor for recognizing and labelling others' emotions. The current findings further support the conclusion that the Stories intervention has merit and warrants further development alongside the Faces intervention.

\section{Strengths and Limitations}

In hindsight, our approach in our previously published analysis which used Spearman correlations to determine whether to include demographic variables as covariates in our main outcomes analysis for this trial had weaknesses. In particular, that approach considered only the linear relationship between potential covariates and the main outcome of interest, and would not account for possible interaction effects that may be present in the data, as indeed have been observed in this subsequent more detailed analysis we have presented here.

We observed a lower proportion of screened women meeting the treatment eligibility criteria than men. (Just $26 \%$ of screened women vs. $38 \%$ of screened men.) It has been suggested that sex may be a protective factor against developing emotion recognition difficulties after brain injury, and the current observation regarding eligibility rates may reflect this [20]. This study also had fairly low statistical power and would not have been able to detect more subtle effects. Future studies would be strengthened through having a larger sample size and having equal numbers of females and males in each treatment arm. There is a paucity of studies examining sex differences in brain research in general [2], and brain injury research in particular. We look forward to further studies adding such analyses to the literature. 
The current study could provide us only with information on sex differences for one of our two interventions, although we know from our previous analysis that men responded positively to the Faces intervention. As women respond positively to the Stories intervention and men do not, it is possible that a similar sex difference is observed for the Faces interventionperhaps women may likewise respond with a greater (or lesser) treatment effect for the Faces intervention than men. Presently, we simply lack information on the response of women to the Faces intervention. By design, the two interventions incorporate a number of shared factors, and it may be that as such interventions are refined further, the evidence will ultimately indicate a combination of their currently unique elements is the most effective approach for both men and women. As we have previously recommended, further research is also needed to examine the broader effects of these treatments on social and emotional capacity in functional contexts $[31,32,37]$. Alongside this, the evidence available is sufficient to recommend thoughtful implementation of these interventions into clinical practice. We are now beginning to see the first independent publications examining implementation of these interventions into practice [e.g., 45].

\section{CONCLUSION}

We concluded in initially reporting on our trial outcomes that we had demonstrated that the Faces intervention was effective at teaching participants, who were on average 11 years postTBI, to better recognize emotions from facial expressions after just three weeks [37]. We now add to that conclusion the further finding that our Stories intervention also appears to be effective in teaching female participants to recognize emotions from facial expressions. This was maintained at three months follow-up, and in the two females retained through to six-month follow-up the effect continued to be observed. In identifying clear sex differences in response to 
the Stories intervention after brain injury, this paper highlights the importance of a greater understanding of how and why emotion recognition difficulties themselves may differ between men and women after traumatic brain injury. 


\section{Acknowledgements}

The authors are indebted to R. C. Gur and colleagues for permission to use their pictures of facial affect in our training program.

\section{Financial Support}

Supported by the National Institute on Disability and Rehabilitation Research (grant no. H133G080043).

\section{Conflict of Interest}

There is a possibility the software package that delivers the intervention described in this study may be released in the future through a publishing company to enable dissemination of the intervention into clinical practice. If this occurs, authors of the article may receive royalty payments associated with sales of the software.

\section{Ethical Standards}

The authors assert that all procedures contributing to this work comply with the ethical standards of the relevant national and institutional committees on human experimentation and with the Helsinki Declaration of 1975, as revised in 2008. 


\section{References}

1. Kret ME, De Gelder B. A review on sex differences in processing emotional signals. Neuropsychologia 2012;50(7):1211-21.

2. McCarthy MM, Arnold AP, Ball GF, Blaustein JD, De Vries GJ. Sex differences in the brain: the not so inconvenient truth. J Neurosci 2012;32(7):2241-7.

3. Stevens JS, Hamann S. Sex differences in brain activation to emotional stimuli: A metaanalysis of neuroimaging studies. Neuropsychologia 2012;50(7):1578-93.

4. Filkowski MM, Olsen RM, Duda B, Wanger TJ, Sabatinelli D. Sex differences in emotional perception: Meta analysis of divergent activation. NeuroImage 2017;147:925-933.

5. Lawrence K, Campbell R, Skuse D. Age, gender, and puberty influence the development of facial emotion recognition. Frontiers in Psychology 2015;6(761).

6. Sullivan S, Campbell A, Hutton SB, Ruffman T. What's good for the goose is not good for the gander: Age and gender differences in scanning emotion faces. The Journals of Gerontology, Series B: Psychological Sciences and Social Sciences 2015.

7. Pavlova MA. Sex and gender affect the social brain: Beyond simplicity. Journal of Neuroscience Research 2017;95(1-2):235-250.

8. McDonald S. Impairments in social cognition following severe traumatic brain injury. Journal of the Interational Neuropsychological Society 2013;19(3):231-246.

9. Babbage DR, Yim J, Zupan B, Neumann D, Tomita MR, Willer B. Meta-analysis of facial affect recognition difficulties after traumatic brain injury. Neuropsychology 2011;25:277285.

10. Radice-Neumann D, Zupan B, Babbage DR, Willer B. Overview of impaired facial affect recognition in persons with traumatic brain injury. Brain injury 2007;21(8):807-816. 
11. Bornhofen C, McDonald S. Emotion perception deficits following traumatic brain injury: A review of the evidence and rationale for intervention. Journal of the International Neuropsychological Society 2008;14(4):511-525.

12. Zupan B, Neumann D, Babbage DR, Willer B. The importance of vocal affect to bimodal processing of emotion: Implications for individuals with traumatic brain injury. Journal of Communication Disorders 2009;42(1):1-17.

13. Zupan B, Neumann D. Affect recognition in traumatic brain injury: Responses to unimodal and multimodal media. The Journal of head trauma rehabilitation 2014;29(4):E1-E12.

14. Bibby $\mathrm{H}, \mathrm{McD}$ onald $\mathrm{S}$. Theory of mind after traumatic brain injury. Neuropsychologia 2005;43(1):99-114.

15. Ferstl EC, Rinck M, von Cramon DY. Emotional and temporal aspects of situation model processing during text comprehension: an event-related fMRI study. J Cogn Neurosci 2005;17(5):724-39.

16. McDonald S, Flanagan S. Social perception deficits after traumatic brain injury: Interaction between emotion recognition, mentalizing ability, and social communication. Neuropsychology 2004;18(3):572-579.

17. Martin-Rodriguez JF, Leon-Carrion J. Theory of mind deficits in patients with acquired brain injury: A quantitative review. Neuropsychologia 2010;48(5):1181-1191.

18. Despins EH, Turkstra LS, Struchen MA, Clark AN. Sex-Based Differences in Perceived Pragmatic Communication Ability of Adults With Traumatic Brain Injury. Arch Phys Med Rehabil 2016;97(2 Suppl):S26-32. 
19. Zupan B, Babbage D, Neumann D, Willer B. Sex differences in emotion recognition and emotional inferencing following severe traumatic brain injury. Brain Impairment 2017;18(1):36-48.

20. Rigon A, Turkstra L, Mutlu B, Duff M. The female advantage: sex as a possible protective factor against emotion recognition impairment following traumatic brain injury. Cognitive, Affective, \& Behavioral Neuroscience 2016;16(5):866-875.

21. Driscoll DM, Dal Monte O, Grafman J. A need for improved training interventions for the remediation of impairments in social functioning following brain injury. Journal of Neurotrauma 2011;28:319-326.

22. Yim J, Babbage DR, Zupan B, Neumann D, Willer B. The relationship between facial affect recognition and cognitive functioning after traumatic brain injury. Brain injury 2013;27(10):1155-1161.

23. Mehrabian A. Silent Messages: Implicit communication of Emotions and Attitudes. Belmont, CA: Wadsworth1981.

24. Zupan B, Babbage D, Neumann D, Willer B. Recognition of facial and vocal affect following traumatic brain injury. Brain Injury 2014;28(8):1087-95.

25. Planalp S. Varieties of cues to emotion in naturally occurring situations. Cognition \& Emotion 1996;10(2):137-154.

26. Planalp S. Communicating emotion in everyday life: Cues, channels, and processes. Handbook of communication and emotion: Research, theory, applications, and contexts 1998:29-48. 
27. Neumann D, Zupan B, Malec JF, Hammond F. Relationships Between Alexithymia, Affect Recognition, and Empathy After Traumatic Brain Injury. The Journal of head trauma rehabilitation 2013; doi: 10.1097/HTR.0b013e31827fb0b5.

28. Knox LaD, J. Long-term ability to interpret facial expression after traumatic brain injuryand its relation to social integration. Brain and Cognition 2009;69:442-49.

29. Milders M, Fuchs S, Crawford JR. Neuropsychological impairments and changes in emotional and social behaviour following severe traumatic brain injury. Journal of Clinical and Experimental Neuropsychology 2003;25(2):157-172.

30. Spikman JM, Milders MV, Visser-Keizer AC, Westerhof-Evers HJ, Herben-Dekker M, van der Naalt J. Deficits in facial emotion recognition indicate behavioral changes and impaired self-awareness after moderate to severe traumatic brain injury. PLoS ONE 2013;8(6):1-7.

31. Biszak AM, Babbage DR. Facial affect recognition difficulties in traumatic brain injury rehabilitation services. Brain Injury 2014;28(1):97-104.

32. Babbage DR. Research priorities for the assessment and treatment of emotion recognition difficulties in brain injury rehabilitation. Critical Reviews in Physical and Rehabilitation Medicine 2014.

33. Radice-Neumann D, Zupan B, Tomita MR, Willer B. Training emotional processing in persons with brain injury. Journal of Head Trauma Rehabilitation 2009;24(5):313-323.

34. McDonald S, Togher L, Tate R, Randall R, English T, Gowland A. A randomised controlled trial evaluating a brief intervention for deficits in recognising emotional prosody following severe ABI. Neuropsychological Rehabilitation 2013;23(2):267-286. 
35. Cassel A, McDonald S, Kelly M, Togher L. Learning from the minds of others: A review of social cognition treatments and their relevance to traumatic brain injury. Neuropsychological Rehabilitation 2016:1-34.

36. Bornhofen $\mathrm{C}, \mathrm{McD}$ onald S. Comparing strategies for treating emotion perception deficits in traumatic brain injury. Journal of Head Trauma Rehabilitation 2008;23(2):103-115.

37. Neumann D, Babbage DR, Zupan B, Willer B. A randomized controlled trial of emotion recognition training after traumatic brain injury. The Journal of Head Trauma Rehabilitation 2014;30(3):E12-E23.

38. Munro BH. Statistical methods for health care research. Lippincott Williams \& Wilkins; 2004.

39. Nowicki S, Jr., Duke MP. Individual-Differences in the Nonverbal-Communication of Affect - the Diagnostic-Analysis of Nonverbal Accuracy Scale. Journal of Nonverbal Behavior 1994;18(1):9-35.

40. McIntire KA, Danforth MM, and Schneider HG. Measuring cue perception: Assessment of reliability and validity. 1997.

41. Spell LA, Frank E. Recognition of nonverbal communication of affect following traumatic brain injury. Journal of Nonverbal Behavior 2000;24:285-300.

42. Nowicki S, Jr., Carton J. The measurement of emotional intensity from facial expressions. J Soc Psychol 1993;133(5):749-50.

43. Zupan B, Neumann D, Babbage DR, Willer B. Exploration of a new tool for assessing emotional inferencing after traumatic brain injury. Brain injury 2015;29(7-8):877-887. 
44. Spikman JM, Boelen DHE, Pijnenborg GHM, Timmerman ME, van der Naalt J, Fasotti L. Who benefits from treatment for executive dysfunction after brain injury? Negative effects of emotion recognition deficits. Neuropsychological Rehabilitation 2013;23(6):824-845.

45. Williamson J, Isaki E. Facial Affect Recognition Training Through Telepractice: Two Case Studies of Individuals with Chronic Traumatic Brain Injury. International Journal of Telerehabilitation 2015;7(1):13. 

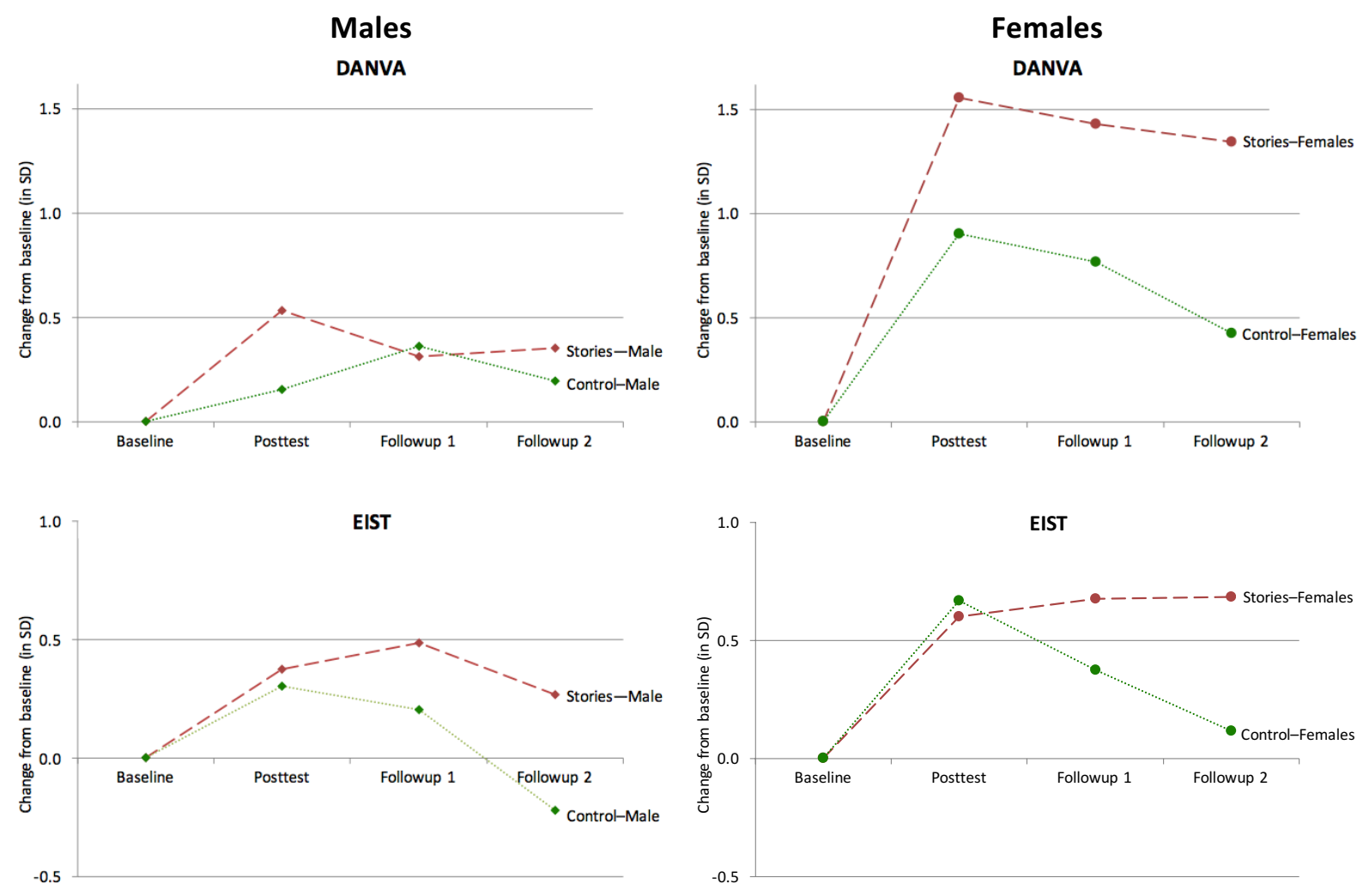

Note: DANVA = Diagnostic Assessment of Nonverbal Accuracy-2 Adult Faces. EIST = Emotional Inference from Stories Test.

Figure 1. Stories intervention vs. control by sex on affect recognition measures. 
Table 1. Comparisons on outcomes for Stories intervention vs. Control by Sex.

\section{DANVA}

\begin{tabular}{lll}
\hline Group & $\mathrm{F} 1,30=5.86 ; \mathrm{P}=.022 ;$ partial $\mathrm{\eta} 2=0.16$ & $\mathrm{~F} 1,42=3.58 ; \mathrm{P}=.113 ;$ partial $\mathrm{\eta} 2=0.08$ \\
Sex & $\mathrm{F} 1,60=6.52 ; \mathrm{P}=.016 ;$ partial $\mathrm{\eta} 2=0.18$ & $\mathrm{~F} 1,84=6.07 ; \mathrm{P}=.058 ;$ partial $\mathrm{\eta} 2=0.12$ \\
Time & $\mathrm{F} 2,60=1.86 ; \mathrm{P}=.164 ;$ partial $\mathrm{\eta} 2=0.06$ & $\mathrm{~F} 2,84=9.41 ; \mathrm{P}=.001 ;$ partial $\mathrm{\eta} 2=0.18$ \\
Group $\times$ Time & $\mathrm{F} 2,60=0.45 ; \mathrm{P}=.641 ;$ partial $\mathrm{\eta} 2=0.02$ & $\mathrm{~F} 2,84=1.35 ; \mathrm{P}=.365 ;$ partial $\mathrm{\eta} 2=0.03$ \\
Sex $\times$ Time & $\mathrm{F} 2,2=0.41 ; \mathrm{P}=.664 ;$ partial $\mathrm{\eta} 2=0.01$ & $\mathrm{~F} 2,2=0.94 ; \mathrm{P}=.459 ;$ partial $\mathrm{\eta} 2=0.02$ \\
Group $\times$ Sex & $\mathrm{F} 1,30=4.71 ; \mathrm{P}=.038 ;$ partial $\mathrm{\eta} 2=0.14$ & $\mathrm{~F} 1,42=0.34 ; \mathrm{P}=.663 ;$ partial $\mathrm{\eta} 2=0.01$ \\
Group $\times$ Sex $\times$ Time & $\mathrm{F} 2,60=0.35 ; \mathrm{P}=.704 ;$ partial $\mathrm{\eta} 2=0.01$ & $\mathrm{~F} 2,84=0.27 ; \mathrm{P}=.785 ;$ partial $\mathrm{\eta} 2=0.01$
\end{tabular}

Note: All analyses examine three post-treatment assessments-post-test, 3 month and 6 month follow-up — with baseline performance as a covariate. DANVA = Diagnostic Assessment of Nonverbal Accuracy-2 Adult Faces. EIST = Emotional Inference from Stories Test. 


\section{Supplemental Online Material}

Table S1. Demographic variables by sex for randomized participants.

\begin{tabular}{lccc} 
& $\begin{array}{c}\text { Male } \\
\text { Mean (SD) }\end{array}$ & $\begin{array}{c}\text { Female } \\
\text { Mean (SD) }\end{array}$ & Difference \\
\hline Age & $41.40(10.63)$ & $38.33(12.69)$ & $\mathrm{F}(1,70)=0.871, \mathrm{P}=.354$ \\
Education, in years & $12.88(2.52)$ & $11.67(1.41)$ & $\mathrm{F}(1,59)=1.967, \mathrm{P}=.166$ \\
Injury severity* & $2.98(0.13)$ & $2.93(0.27)$ & $\mathrm{F}(1,68)=0.874, \mathrm{P}=.353$ \\
Time since injury, in years & $10.84(8.74)$ & $8.44(6.37)$ & $\mathrm{F}(1,70)=1.178, \mathrm{P}=.281$ \\
\hline
\end{tabular}

Note: * Injury severity coded as $1=$ Mild, $2=$ Moderate, $3=$ Severe. All participants experienced moderate or severe injuries. 
Table S2. Outcome measure means and standard deviations at baseline, post-test, and follow-up by sex for Stories and Control interventions.

\begin{tabular}{lcccc}
\hline & $\begin{array}{c}\text { Baseline } \\
\text { Mean (SD) }\end{array}$ & $\begin{array}{c}\text { Post-test } \\
\text { Mean (SD) }\end{array}$ & $\begin{array}{c}\text { Follow-up 1 } \\
\text { Mean (SD) }\end{array}$ & $\begin{array}{c}\text { Follow-up 2 } \\
\text { Mean (SD) }\end{array}$ \\
\hline $\begin{array}{l}\text { Diagnostic Assessment of Nonverbal Accuracy-2 } \\
\text { Males }\end{array}$ & $-1.75(0.77)$ & $-1.08(1.13)$ & $-1.36(1.56)$ & $-1.31(1.23)$ \\
Stories & $-2.21(0.87)$ & $-2.02(1.29)$ & $-1.76(1.41)$ & $-1.97(1.11)$ \\
Control & & & & \\
$\begin{array}{l}\text { Females } \\
\text { Stories }\end{array}$ & $-1.97(0.72)$ & $-0.03(0.73)$ & $-0.19(0.92)$ & $-0.29(1.02)$ \\
Control & $-2.21(0.98)$ & $-1.08(1.57)$ & $-1.25(1.35)$ & $-1.68(1.79)$ \\
\hline $\begin{array}{l}\text { Emotional Inference from Stories } \\
\text { Males } \\
\text { Stories }\end{array}$ & & & & \\
Control & $7.54(2.16)$ & $8.54(2.32)$ & $8.83(2.51)$ & $8.25(3.17)$ \\
Females & $7.50(2.03)$ & $8.31(1.54)$ & $8.04(2.60)$ & $6.91(3.04)$ \\
Stories & & & & \\
Control & & & & \\
& $9.20(2.56)$ & $10.80(1.30)$ & $11.00(1.23)$ & $11.02(2.11)$ \\
\hline & $8.31(2.63)$ & $10.09(2.02)$ & $9.31(2.82)$ & $8.62(3.27)$ \\
\hline
\end{tabular}

Note: Table displays pooled means and standard deviations across 50 imputation datasets.

DANVA Faces scores are $\mathrm{Z}$ scores. 